\title{
Lossio, 0.; Peretti, G. (Comp.) (2020). Geografía de Santa Fe: transformaciones recientes del territorio provincial.
}

\author{
Santa Fe: Ediciones UNL, 131 pp.
}

MARÍA BELÉN WILLINER* Y LAUTARO MÉNDEZ**

*Facultad de Humanidades y Ciencias, Universidad Nacional del Litoral, Argentina.

${ }^{* *}$ Facultad de Ciencias Agrarias, Universidad Nacional del Litoral, Argentina.

belenwilliner@gmail.com; lautaroamendez@gmail.com

\section{Introducción}

Los que somos recientes egresados de la carrera de Geografía hemos escuchado, leído y discutido diferentes artículos y libros de los y las geógrafas Cervera, Manzi, Gallardo y D’Angelo sobre Geografía de Santa Fe. No obstante, dichas obras hablan de un territorio santafesino correspondiente a otros tiempos, con otras problemáticas y procesos espaciales diferentes a los actuales. Su metodología y epistemología utilizada fueron descriptivas desde un abordaje un tanto positivista. Eran tiempos donde este tipo de Geografía tenía gran presencia en la Academia, es decir, un formato "enciclopedista", de recopilación de datos e información.

Sin embargo, desde 1980 aproximadamente, la Geografía ha sido enriquecida por aportes de los estudios culturales concibiendo al espacio geográfico como producto y productor de relaciones sociales a lo largo del tiempo. Dicho espacio, que involucra tanto lo material como lo simbólico, actúa de soporte, sostén y condición de las relaciones y de los procesos sociales que generan objetos que se manifiestan en el espacio. Ese espacio deja de considerarse como contenedor de elementos y pasa a ser un producto-productor de relaciones sociales; el espacio nos construye al mismo tiempo que lo construimos a él. Ante esta necesidad de repensar nuestro objeto de análisis, los estudios geográficos optaron por otros enfoques, marcos teóricos y epistemológicos.

La idea original del libro "Geografia de Santa Fe: transformaciones recientes del territorio provincial", compilado por Oscar Lossio y Gustavo 
Peretti, nació a partir de demandas de profesores que se desempeñan en el nivel secundario y que han participado de las diferentes Jornadas de Geografía organizadas por el Departamento de Geografía de la Facultad de Humanidades y Ciencias (FHUC) en las que se puso de manifiesto la necesidad de contar con recursos actualizados para pensar y planificar nuestras clases sobre geografía de Santa Fe. Ahora bien, cabe destacar que no es un libro destinado al público adolescente, sino que es un material que puede ser utilizado como insumo docente; además, tal como lo indica en el prólogo María Luisa D’Angelo, puede trascender las áreas de la docencia y la investigación para convertirse en una herramienta para aquellos que toman decisiones políticas en el territorio provincial.

Esta obra presenta una fresca y renovada mirada de la geografía santafesina a partir de diferentes trabajos de docentes-investigadores integrantes del Departamento de Geografía de la FHUC. Es de destacar que la publicación puede descargarse libremente en la biblioteca virtual de la UNL ${ }^{\mathrm{I}}$. Además, fue publicitado en diferentes redes sociales con la finalidad de extender su divulgación como también una presentación del mismo en una videoconferencia.

En cada uno de los seis capítulos que estructuran a esta obra, las y los autores analizan diversas problemáticas y transformaciones territoriales de la provincia de Santa Fe, las cuales son derivados de la reestructuración misma del sistema capitalista, provocando que los espacios rurales y urbanos se redefinan o muten junto a las relaciones que los conforman.

De acuerdo con el abordaje teórico y metodológico, las y los autores se posicionan con un estilo y formas de organización de la escritura totalmente diferentes. Los capítulos tienen un orden particular, lo cual se hace interesante recuperar, ya que organizaron el libro de acuerdo a escalas geográficas interrelacionadas desde lo rural a lo urbano, haciendo mucho hincapié en los espacios de continuum urbano-rural. Es así que el primer capítulo da cuenta de procesos socioterritoriales en el distrito rural de Matilde (provincia de Santa Fe), el segundo, por su parte, trata de la construcción del cinturón hortícola en espacios rururbanos y periurbanos por parte de migrantes bolivianos; el tercero, sobre el área metropolitana de Santa Fe y los procesos de contra y sub urbanización; el cuarto sobre la reconfiguración de la población urbana en la

1 Disponible en: https://bibliotecavirtual.unl.edu.ar:8443/handle/11185/5572 
provincia; el quinto capítulo es sobre ciudades intermedias y sus posibilidades como espacios de desarrollo territorial y por último, el sexto, las transformaciones del sector portuario en el municipio de Santa Fe.

\section{Problemáticas territoriales santafesinas contemporáneas: análisis de la obra}

El primer capítulo se titula "Transformaciones en las actividades agropecuarias en el distrito Matilde en el periodo 1990-2010. Una investigación desde la Geografía constructivista" de Oscar Lossio. El autor analiza cómo a partir de la reestructuración capitalista de los 70, existió una transformación productiva en la actividad tambera a los recientes procesos de agriculturización y sojización en el distrito Matilde. El marco epistemológico de la geografía constructivista "busca la comprensión del espacio desde la perspectiva del sujeto, es decir, de quién habita el lugar, reconociendo un rol central al lenguaje, incluso, como creador de realidad" (Lossio, 2020:I5), por lo que el autor decide centrarse en las metodologías cualitativas, principalmente en la entrevista en profundidad, para construir a su sujeto de investigación. Las narrativas de los productores entrevistados manifestaron diversos significados y acciones que transformaron el espacio rural, muchas veces entrando en conflicto con las tradiciones familiares del distrito. El texto hace un recorrido sobre el nacimiento de la producción lechera en la región y si bien el tambo conformaba parte de la identidad de los productores, en los 90, y con el correr de las generaciones y la menor rentabilidad de la actividad, la abandonaron para abocarse a la agricultura. Un capítulo que, a nuestro criterio, sirve para correlacionar con otros distritos de la provincia de Santa $\mathrm{Fe}$, donde el contexto a nivel nacional e internacional hizo que la actividad sojera cobre mayor relevancia frente a la tambera.

El segundo capítulo se denomina "La migración boliviana al cinturón horticola santafesino: aportes teóricos-conceptuales para su análisis socioespacial" de Mariela Demarchi. El texto desarrolla un interesante marco teórico sobre la construcción del espacio y las escalas como red y relaciones para así poder comprender a las migraciones desde su complejidad y cómo estas interfieren en el espacio. La autora interpreta a las migraciones desde la teoría "de las Redes Sociales" concentrándose principalmente en los movimientos poblacionales desde Tarija (Bolivia) al 
cinturón hortícola ubicado al norte de la ciudad de Santa Fe. A través del trabajo de campo y entrevistas a los migrantes, se ha podido llegar a comprender sus experiencias sociales y espaciales de su vida cotidiana y agruparlas en:

I) La cotidianeidad laboral de la comunidad; 2) Las experiencias simbólicas «vividas»; 3) La revitalización de los vínculos sociales pasados y presente y 4) Los significados y percepciones: la acción y la valorización del «logro» obtenido (Demarchi, 2020:52).

El tercer capítulo denominado "El fenómeno de la contraurbanización: perspectiva teórica y estudio de caso en el área metropolitana de Santa Fe", de María Mercedes Cardoso, da cuenta de los procesos de las transformaciones espaciales que han sufrido los espacios urbanos en los últimos años, enfocándose particularmente en el área metropolitana de Santa Fe. La autora observó que se hicieron presente dos procesos en simultáneo y que no son contrapuestos entre sí: por un lado, la suburbanización y por el otro, la contraurbanización. El primero lo entiende como "el crecimiento poblacional y económico de las áreas suburbanas, inmediatas a las ciudades" mientras que al segundo como "el crecimiento poblacional y económico que se da en las áreas rururbanas, rurales remotas y pequeñas ciudades de los alrededores" (Cardoso, 2020:64), es decir, el hecho que la mancha urbana se expanda sobre los espacios que anteriormente eran rurales, hoy periurbanos o rurubanos, daría cuenta de conflictos, tensiones y resistencias por el uso del suelo como así también se demandaría nuevas regulaciones de ordenamiento territorial. Para su estudio de caso en el Área Metropolitana de Santa Fe, identifica ciertos procesos de contraurbanización, ya que algunas localidades que componen al "Gran Santa Fe" tendrían un importante crecimiento urbano frente a la ciudad capital provincial, es decir, esta aparente desconcentración de la población ya no sería relativa sino absoluta. Por otro lado, el proceso de suburbanización, explicaría el crecimiento de los espacios anteriormente rurales, hoy rururbanos, en el norte del municipio de Santa Fe. Además de ello, la autora hace mención a las diferentes teorías que explicarían las relaciones campo-ciudad las cuales han cambiado a lo largo del tiempo. Por último, Cardoso concluye que a medida que las grandes ciudades se desconcentran, producto de diferentes problemáticas urbanas, las localidades pequeñas o medianas junto a áreas rururbanas y rurales estarían 
creciendo de manera absoluta, tal como lo indican los diversos Censos Nacionales de Población, Hogares y Viviendas del período I99I al 2010.

Con respecto al capítulo cuatro de los autores Javier Gómez y Gustavo Peretti, denominado "Distribución poblacional y urbanización en la provincia de Santa Fe. Período I895-2010", se analiza la historicidad de la distribución de la población durante el período censal I895-20Io. En dicho período, se observa un proceso de primacía y macrocefalia urbana, donde las ciudades de Rosario y Santa Fe concentran más del $98 \%$ de la población urbana ${ }^{2}$ de la provincia. No obstante, los últimos censos nacionales reflejan ciertos cambios demográficos en tales localidades lo cual podría indicar un "retroceso" o una disminución de la macrocefalia y primacía urbana santafesina. En palabras de los autores: "la macrocefalia, si bien disminuyó entre 1947 y 20I0, lo ha hecho a un ritmo menos marcado, por lo cual se puede concluir que, si bien la representatividad poblacional del Gran Rosario ha disminuido, su gravitación continúa siendo marcada" (Gómez y Peretti, 2020:94). Es decir, la importancia regional de ambas localidades no disminuyó sino su población absoluta frente a otros distritos lindantes a las mismas. Por citar algunos ejemplos, tal como lo observa Cardoso en el capítulo tres, distritos que componen al Área Metropolitana de Santa Fe, estarían creciendo en cantidad de población frente a la capital. Para concluir, los autores indican que desde 1895 a 2010 la cantidad de localidades urbanas ha crecido con una variación de $200 \%$, sobre todo en la región centro de la provincia, donde el proceso de colonización impactó con mayor magnitud con respecto a otras regiones.

En el capítulo cinco, bajo el título de "Ciudades intermedias y desarrollo territorial. Una mirada hacia la provincia de Santa Fe" de Carina Davies, la autora describe la relación que habría entre ciudades intermedias y su desarrollo territorial. Para ello, Davies realiza un estudio de caso de dos localidades: Venado Tuerto y Reconquista, ubicadas en el sur y norte provincial respectivamente. En el contenido del capítulo, se conceptualiza al desarrollo territorial como algo no exclusivo en lo económico sino:

2 Se entiende como población urbana a aquellos distritos cuya población supera los 2000 habitantes (INDEC). 
Que también debe ser capaz de desplegar sus componentes sociales (bienestar), ambientales (sostenibilidad), políticos (gobernabilidad y participación local), culturales (defensa de la identidad y el patrimonio) y geográficos (ordenación del territorio), persiguiendo el objetivo primario de alcanzar mayores niveles de calidad de vida al interior de las sociedades en las cuales se despliegan estas políticas (Méndez en Davies, 2020:98).

De acuerdo con este concepto propuesto por el autor espańol, la autora en cuestión lo relaciona con el de ciudades intermedias, considerando estos núcleos urbanos no solo por un umbral poblacional ${ }^{3}$ sino por su "capacidad de convertirse en centros de recursos específicos para ofrecer una amplia variedad de servicios y se difunda el conocimiento y dinamismo, permitiendo equilibrar el territorio frente a las macrocefalias metropolitanas" (Davies, 2020:98). Por lo tanto, Davies, realiza un trabajo comparativo de estas dos ciudades intermedias nombradas anteriormente las cuales, a su vez, son "cabeceras" del Plan Estratégico Provincial (PEP) puesto en marcha a partir del año 2008, que divide a la provincia en cinco regiones con su correspondiente núcleo nodal. Estas dos ciudades son sede de Agencias de Desarrollo Regional (ADR), instituciones que forman parte del Plan Estratégico Territorial (PET), siendo ambos elementos impulsados desde el Estado nacional. La autora concluye que tanto Reconquista como Venado Tuerto, como ciudades intermedias, cuentan con una importante red de entidades con un rol clave: fomentar el desarrollo territorial en otras áreas urbanas, restando de alguna forma, la marcada macrocefalia del sistema urbano argentino y santafesino, tal como lo explican Gómez y Peretti en el capítulo cuatro.

El último capítulo se denomina "Procesos de transformación en el sector del puerto de la ciudad de Santa Fe de la Vera Cruz. ¿Un nuevo espacio público?" cuyas autoras son Norma Finelli y María Mercedes Cardoso. A lo largo del capítulo podemos interpretar cuáles fueron las principales transformaciones de ese sector icónico de la ciudad, desde la época colonial (cuando se lo declaró "puerto preciso" en I739); su función durante la etapa de la economía primaria agroexportadora hasta llegar a los finales de los 70, cuando inicia el declive de la actividad portuaria. Es así que, desde esa década, el distrito del puerto se ha visto atravesado por los diferentes procesos de reconversión, rehabilitación y marketing

3 Dicho umbral es entre los 50000 y 150000 habitantes. 
urbano en los que se ha transformado el espacio debido a la inversión pública y privada, y que actualmente se observan diversas actividades comerciales, recreativas y turísticas que lo desvinculan de su función portuaria. Hacia el final del capítulo, las autoras se preguntan si en el proceso de reconversión del puerto se ha perdido espacio público y si contribuyó a una mayor fragmentación urbana.

\section{Conclusiones}

Como se ha indicado al inicio de la reseña, consideramos que el libro es un importante aporte al estudio y análisis de las principales transformaciones territoriales que han ocurrido en la provincia desde los 70 a la actualidad. Es, sin duda, un material que será de suma importancia para la formación de futuros geógrafos, como así también puede proporcionar un interesante marco teórico y conceptual para abordar nuevas problemáticas de las Ciencias Sociales.

No obstante, consideramos que al interior de los capítulos se visualiza escaso material cartográfico que apoye el discurso de los autores lo que podría ser adecuado para comprender aún mejor los procesos y problemáticas territoriales desde las diferentes escalas geográficas utilizadas. Por otro lado, observamos que algunas problemáticas no han sido abordadas, tales como: los procesos de mercantilización de la naturaleza, problemas ambientales en el espacio periurbano, la fragmentación urbana, construcción social del riesgo en diferentes localidades como así también los conflictos en torno a los usos de los espacios isleños, pero consideramos que pueden dar lugar a futuras investigaciones y publicaciones.

Para finalizar, creemos que el libro es un interesante aporte y que las acciones de investigación y divulgación de la información deberían ser prácticas más comunes entre los geógrafos, como así también la construcción de nexos y vinculaciones entre los ámbitos académicos, los educativos y los de decisión política. 


\section{Referencias bibliográficas}

Cardoso, M. (2020). El fenómeno de la contraurbanización: perspectiva teórica y estudio de caso en el área metropolitana de Santa Fe. En Lossio, O. y Peretti, G. (Comp.) Geografía de Santa Fe: transformaciones recientes del territorio provincial. Santa Fe: Ed. UNL.

Davies, C. (2020). Ciudades intermedias y desarrollo territorial. Una mirada hacia la provincia de Santa Fe. En Lossio, O. y Peretti, G. (Comp.) Geografía de Santa Fe: transformaciones recientes del territorio provincial. Santa Fe: Ed. UNL.

Demarchi, M. (2020). La migración boliviana al cinturón hortícola santafesino: aportes teóricos-conceptuales para su análisis socioespacial. En Lossio, O. y Peretti, G. (Comp.) Geografía de Santa Fe: transformaciones recientes del territorio provincial. Santa Fe: Ed. UNL.

Gómez, J. y Peretti, G. (2020). Distribución poblacional y urbanización en la provincia de Santa Fe. Período I895-2010. En Lossio, O. y Peretti, G. (Comp.) Geografia de Santa Fe: transformaciones recientes del territorio provincial. Santa Fe: Ed. UNL.

Lossio, O. (2020). Transformaciones en las actividades agropecuarias en el distrito Matilde en el período I990-20ı0. Una investigación desde la Geografía constructivista. En Lossio, O. y Peretti, G. (Comp.) Geografía de Santa Fe: transformaciones recientes del territorio provincial. Santa Fe: Ed. UNL. 